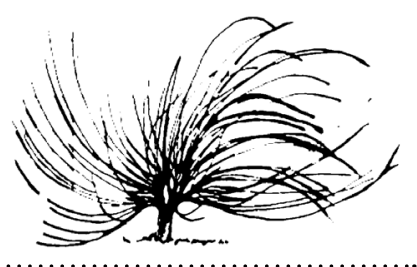

\title{
Deporte, violencia y masculinidad en Norbert Elias y Eric Dunning. Una lectura orientada al ámbito escolar ${ }^{1}$
}

\author{
Nicolás Patierno ${ }^{2}$ \\ Consejo Nacional de Investigaciones Científicas y Técnicas (CONICET) \\ Argentina \\ nicolaspatierno@gmail.com \\ Pablo Kopelovich ${ }^{3}$ \\ Consejo Nacional de Investigaciones Científicas y Técnicas (CONICET) \\ Argentina \\ kopelovichp@gmail.com
}

\begin{abstract}
Resumen
El presente ensayo tiene como objetivo analizar el deporte y la violencia con especial referencia al fútbol, desnaturalizando aquellas representaciones por las que se presume que esta práctica puede ser considerada "agresiva" en sí misma. En contraposición, y siguiendo los aportes de Norbert Elias y Eric Dunning, entendemos este juego y la violencia como categorías antagónicas, ya que, en
\end{abstract}

Recibido: 20 de enero de 2020. Aprobado: 2 de setiembre de 2020.

http://dx.doi.org/10.15359/rep.16-1.1

1 El presente ensayo es el resultado del encuentro, el intercambio y la profundización de dos investigaciones conducentes a la realización de dos tesis. La correspondiente a Nicolás Patierno se titula "La violencia (escolar) en cuestión. Un estudio en escuelas secundarias públicas de la ciudad de La Plata" y fue presentada para optar al título de Doctor en Ciencias de la Educación, Universidad Nacional de La Plata, Argentina, año 2019. La realizada por Pablo Kopelovich se titula "Educación física y escuela secundaria: sentidos y prácticas en torno a la enseñanza de la asignatura en un colegio de la provincia de Buenos Aires” y fue presentada para optar al título de Magíster en Ciencias Sociales con orientación en Educación, Facultad Latinoamericana de Ciencias Sociales, Argentina, año 2018.

2 Profesor en Educación Física (Universidad Nacional de La Plata), Magíster en Educación Corporal (Universidad Nacional de La Plata) y Doctor en Ciencias de la Educación (Universidad Nacional de La Plata)

3 Profesor y Licenciado en Educación Física (Universidad Nacional de La Plata), Magíster en Ciencias Sociales con orientación en Educación (Facultad Latinoamericana de Ciencias Sociales). 
explícito acuerdo con la hipótesis de estos autores, uno de los propósitos originarios del deporte es la mitigación de la violencia. Intentaremos desandar los caminos por los que se ha estigmatizado al fútbol desalentando su práctica en algunos ámbitos educativos; complementariamente, estudiaremos la influencia de algunas categorías constitutivas de la problemática planteada, tales como: masculinidad, hinchada, "aguante" y sensacionalismo mediático, entre otras. Siguiendo esta línea, la conclusión invita a recuperar el valor educativo del deporte, más precisamente, su relevancia en los procesos de socialización y el reconocimiento de las reglas.

Palabras clave: deporte, violencia, fútbol, masculinidad, enseñanza.

\begin{abstract}
This paper aims to analyze sport and violence with special reference to football, denaturing those representations by which it is presumed that this practice can be considered "violent" in itself. In contrast, and following the contributions of Norbert Elias and Eric Dunning, we understand football and violence as antagonistic categories, since in explicit agreement with the hypothesis of these authors, one of the original purposes of sport is the mitigation of violence. We will try to retrace the paths by which football has been stigmatized by discouraging its practice in some educational fields. Complementarily, we will study the influence of some constitutive categories of the problem posed, such as masculinity, fanaticism, "football stamina" and media sensationalism, among others. Following this line, the conclusion invites us to recover the educational value of sport, more precisely, its relevance in the processes of socialization and recognition of the rules.
\end{abstract}

Keywords: sport, violence, soccer, masculinity, teaching 


\section{Introducción}

$\mathrm{E}$

n la mayoría de los currículos escolares y los proyectos educativos pensados para la enseñanza secundaria en los que se incluye el deporte, el argumento de su incorporación siempre suele ser el mismo: su valor socializador. El propio Diseño Curricular para la Educación Secundaria de la Provincia de Buenos Aires (Argentina) contempla la relevancia del deporte como un medio de socialización, arguyendo que en este:

Se despliegan las capacidades sociales y relacionales que permiten la construcción de normas y reglas, el desarrollo de la solidaridad, la cooperación, el respeto, la ejercitación de su responsabilidad y el fortalecimiento de vínculos con los demás. (Dirección General de Cultura y Educación de la Provincia de Buenos Aires, 2010, p. 28)

Las causas de esta vinculación pueden hallarse en la génesis de los deportes, además de constituirse como de distinción social, su creación fue pensada para reglamentar ciertos juegos populares y comportamientos inicialmente, asociados a la violencia y el descontrol. De esta manera, actividades medievales como el knappan, caracterizadas por una lucha de todos contra todos (donde la violencia física formaba parte de ella), comienzan a mutar hacia prácticas más regladas y con menos contacto cuerpo a cuerpo.

Resumidamente, el knappan es un juego popular de la Edad Media sin límite de jugadores, ni espacio, y con reglas orales en el que se desarrolla una lucha generalizada de todos contra todos (Dunning, 1992). Con un marcado contenido de lo que Dunning (1992), denomina violencia afectiva, los pobladores solían utilizar el evento como una purga para descargar físicamente los resentimientos acumulados hacia familiares, vecinos, etc.

El análisis de esta práctica no es azaroso, su vinculación directa con la Edad Media sirve de respaldo a los estudios de Elias y Dunning (1992), sobre el avance de la civilización y la limitación de acciones violentas en las actividades recreativas. Poco a poco, el juego mencionado introdujo restricciones sobre la violencia física, y sus reglas 
fueron explicitadas de manera escrita, dando paso a lo que hoy conocemos como rugby (Barbero, 1993).

En este contexto, el deporte puede ser considerado como:

[...] una actividad de grupo organizada y centrada en la competición entre al menos dos partes. Exige algún tipo de ejercicio o esfuerzo físico. El enfrentamiento se realiza siguiendo reglas conocidas, incluidas -en los casos en que se permite el uso de la fuerza física- las que definen los límites de violencia permitidos. (Elias,1992, p. 190)

En este sentido, la codificación y la institucionalización de los comportamientos considerados "brutales" fue muy útil para el proceso civilizatorio encabezado por los países más industrializados, que tiene su origen a mediados del siglo XIX (y se expande globalmente hasta nuestros días).

Si bien podría considerarse que la función original del deporte fue la sublimación y el apaciguamiento de los jóvenes de las clases burguesas que asistían a las public schools (Barbero, 1993), este fue tan solo uno de los múltiples usos que posteriormente, se le atribuirían a lo largo del siglo XX (Hernández y Carballo, 2002).

El deporte sirvió -y sirve- para la distinción de clases, para el control de las masas, para la comercialización de productos, para la producción de espectáculos y otras tantas utilidades que hoy lo convierten en mucho más que un pasatiempo ocasional. De todos los atributos que comúnmente se le suelen asignar -y al mismo tiempo se le critican-, aquí se centrará la atención en las funciones socializadoras dentro del ámbito educativo.

Como veremos a lo largo de este trabajo, la aceptación del reglamento, el respeto por los demás participantes y la aprobación del arbitraje requieren de un minucioso y progresivo trabajo de enseñanza, dirigido a la renuncia creciente al individualismo y al empleo de la violencia. En pocas palabras, podríamos afirmar que la violencia es siempre enemiga del deporte: en el desarrollo de cualquier especialidad -cuando es debidamente reglamentada y fiscalizada-, cualquier exceso en el uso de la fuerza constituye una falta (situación que representa una ventaja para el oponente). 
Los deportes de combate no son una excepción: si bien para el observador externo un golpe de puño o una patada pueden representar, a simple vista, una acción violenta, al estar normalizadas por un reglamento no pueden ser caracterizadas bajo esta categoría. El eje de este tipo de entretenimientos está en la posibilidad de entablar una batalla mimética en la que los contactos están previstos y se regulan a partir de un código predeterminado, con lo que no sería pertinente hablar de violencia en tales circunstancias.

De acuerdo con Garriga, "un mismo actor puede estar inserto en una trama relacional que impugne prácticas que él considere violentas $\mathrm{y}$, al mismo tiempo, sea parte de acciones que otros consideran como violentas" (2016, p. 14). Sin embargo, algunas prácticas de combate específicamente contemporáneas (como las artes marciales mixtas) introducen una controversial y delicada espectacularización del knock out.

Si bien este tipo de actividades podría caracterizarse como uno de los máximos exponentes actuales del deporte-espectáculo, incluso admitiendo restricciones penalizadas en otros deportes de contacto como las inmovilizaciones, el sangrado o los golpes bajos-, en tanto los competidores no se encuentren coaccionados bajo ninguna circunstancia, no sería preciso caracterizarlas como violentas.

\section{El deporte y la violencia como categorías antagónicas}

Los estudios de Elias (1992), centrados en el deporte como parámetro de medición de ciertas conductas tendientes al autocontrol, constituyen una fuente de información ineludible en la temática. El sociólogo alemán caracteriza las sociedades posrevolucionarias como constitutivas de un proceso civilizador, el cual se evidencia, entre otras prácticas, en los modos en que se regulan y ejecutan los deportes.

A partir de un minucioso análisis sobre el devenir de la caza de zorros en Inglaterra durante los siglos XVII y XVIII, Elias (1992), halla que, a medida que la sociedad inglesa supera los enfrentamientos armados entre bandos que rivalizan por el mando del país, se inicia un proceso de pacificación evidente en las características figuracionales de los deportes ${ }^{4}$. Confluyen dos procesos específicamente modernos:

4 Bajo la idea de figuraciones, Elias propone analizar los deportes atendiendo tanto al desarrollo de las reglas como a las relaciones de interdependencia que se tejen entre los individuos (arguyendo que estas categorías no pueden analizarse de manera aislada). A partir de sus estudios enfocados en el pasaje de la sociedad inglesa de la Edad Media al siglo XVIII, Elias fue 
la constitución del yo -o, en otras palabras, la configuración del ideal de hombre autorregulado- $y$ una progresiva desaparición de comportamientos que remitirían a cierta barbarie o salvajismo.

Podemos hallar un ejemplo de lo mencionado en la matanza de animales: mientras que en la Edad Media se trataba de una práctica socialmente aceptada, hoy las actividades que implican la muerte de ellos poseen una connotación negativa, generan descontento social y se hallan prácticamente desaparecidas.

Elias (1992), contextualiza la Inglaterra del siglo XVIII -sobre todo los primeros años-como una época de conflictos y luchas armadas por el poder en las que no se evidenciaba la posibilidad de una rápida salida pacífica. A partir de allí, introduce el análisis de la caza de zorros como modelo empírico para desarrollar algunas de las características estructurales de los deportes.

En un proceso que va del desorden a la conformación del parlamento inglés y la consolidación de una sociedad industrial, la caza de zorros es una especie de termómetro empleado por Elias (1992), para medir su desarrollo: dado que esta actividad también sufrió cambios determinantes en su reglamentación (pasando de ser una actividad de supervivencia en la Edad Media a un refinado entretenimiento de las clases altas en la época victoriana), dicho autor afirma que ambos aspectos se hallan influenciados mutuamente.

En el comienzo de Un ensayo sobre el deporte y la violencia, el sociólogo ratifica la existencia de un paralelismo entre "la difusión desde Inglaterra de modelos industriales de producción, organización y trabajo, y [...] la difusión de actividades de tiempo libre del tipo conocido como «deporte»" (1992, p. 185).

Centrando la atención en la cuestión de la violencia, el sociólogo advierte que, mientras que en un comienzo la caza de zorros era llevada a cabo directamente por las personas, a lo largo del siglo XVIII se incluye la utilización de perros para esta tarea. Este pasaje de matar con las propias manos a dejar que los mastines lo hagan y experimentar placer durante el proceso, evidenciaría cierto progreso hacia la civilización. En palabras del propio Elias: 
La dirección de los cambios experimentados en la manera de cazar, si se contrasta el ritual inglés de la caza de zorros con anteriores formas de caza, muestra muy claramente la dirección general de un empuje civilizador. El aumento de las restricciones sobre el empleo de la fuerza física y en particular sobre el hecho de matar y, como expresión de estas restricciones, el desplazamiento del placer experimentado cometiendo actos violentos al placer experimentado viendo cometer actos de violencia, pueden observarse como síntomas de un empuje civilizador en muchas otras esferas de la actividad humana. (1992, pp. 199-200)

El placer de la caza de zorros, por lo tanto, ya no descansaría únicamente en la matanza, sino en la extensión del proceso de cacería (mediante la implementación de una serie de limitaciones cada vez más complejas, hoy conocidas como reglas). La muerte rápida del animal a punta de cañón significaría una culminación veloz del proceso; en cambio, las restricciones propuestas tenían como objetivo extender el goce devenido de la persecución.

De esta manera, se establece un equilibrio de tensiones entre el proceso de persecución y la muerte del animal, lo que aseguraría la experimentación de una tensión-emoción agradable. "La violencia se practicaba con animales en lugar de seres humanos, $[\ldots]$ las personas disfrutaban con la muerte del animal cazado de una forma indirecta, como observadores" (Elias, 1992, p. 203).

El desplazamiento de cazar por supervivencia -para obtener alimentos- a hacerlo por deporte -para adquirir placer es: "una emoción agradable derivada de la batalla mimética" (Elias, 1992, p. 202)- es un pasaje que se alinearía con la constitución de una sociedad más civilizada.

En este proceso, el sociólogo introduce dos categorías: un avance en el umbral de la sensibilidad y la formación de la conciencia. La primera de ellas hace referencia al crecimiento de las prohibiciones sociales contra el hecho de matar y de ver cómo otros lo hacen. Es por este motivo que el sociólogo encuentra la prohibición de la participación humana en la matanza de zorros como una acción tendiente a la disminución de la violencia.

Dunning (1992), se va a pronunciar a favor de esta hipótesis arguyendo que estamos en presencia de un "declive en el anhelo de atacar 
[...]. Esto ha llevado consigo, primero, un descenso en el umbral de rechazo al derramamiento de sangre y otras manifestaciones directas de la violencia física" (1992, p. 275).

La segunda categoría se desprende directamente de la primera y se centra en la inclusión de animales en el proceso de caza. Si bien hoy en día esta práctica resultaría bastante controversial, la inserción de mastines representaba por entonces "la irradiación del sentimiento más allá del objetivo inicial" (Elias, 1992, p. 203).

El distanciamiento en el acto de dar muerte y el empleo de otros animales para ejecutar la presa significan, para el autor, una especie de ejercicio de la conciencia que aleja al humano de un comportamiento primitivo. Para Dunning, a su vez, se trata de "la interiorización de un tabú más estricto sobre la violencia [...]. Consiguientemente tienden a brotar sentimientos de culpa cada vez que este tabú es violado" (1992, p. 275).

En este contexto de creciente civilización, el deporte representa una especie de invento social que resolvería dos problemas derivados de vivir en una sociedad cada vez más organizada. Por un lado, determina qué hacer con el tiempo libre, y por otro, canaliza positivamente, la tensión derivada de estar bajo ciertas restricciones.

\section{Deporte, violencia y educación}

De acuerdo con lo desarrollado hasta aquí (y pese a que el análisis de Elias -1981- está más enfocado al proceso de la civilización y la deportivización que al estudio específico de la violencia) $)^{5}$, esperaríamos una distinción clara entre ella y el deporte, que reconozca en el primero una potencia socializadora que la educación no debería desatender. Sin embargo, Crisorio (2010), advierte que el deporte es -con frecuenciaacusado de "violento", conflictivo o tendiente al descontrol.

La asociación entre deporte y violencia remite a una serie representaciones provenientes de otros campos "que tienden a condenarlos

5 En la conferencia publicada bajo el título Civilización y violencia, Elias menciona algunas precisiones respecto de su interpretación, estas pueden ayudarnos a entender mejor su perspectiva: "normalmente, a la hora de investigar el problema de la violencia se sigue un enfoque erróneo. Se pregunta, por ejemplo, cómo es posible que los seres humanos cometan asesinatos [...]. Cuando en realidad la pregunta debería ser enfocada de otra manera, de manera opuesta: ¿cómo es posible que tantas personas convivan de manera - relativamente- tan pacífica?” (1981, p. 65). Siguiendo esta línea, resumidamente podría decirse que, conforme el proceso de la civilización avance, la violencia debería ocupar un lugar cada vez menos relevante en las sociedades contemporáneas. 
en cuanto actividades competitivas, que llevan en sí mismas la rivalidad y la agresión, e incitan a la violencia en los propios deportistas y en los espectadores" (Crisorio, 2010, p. 190).

El problema aquí es que la competitividad y las utilidades económicas que rodean el deporte-espectáculo convierten a este tipo de prácticas en algo mucho más complejo e intrincado que una simple lucha de carácter mimético, inicialmente, pensada para sublimar tensiones. Por lo tanto, se vuelve imprescindible discernir entre deporte-educación y deporte-espectáculo, entre una actividad pensada para la socialización de las nuevas generaciones y otra ligada a la espectacularización y la obtención de ganancias ${ }^{6}$.

Aquí es pertinente atender a los aportes de Dunning, quien sostiene la hipótesis de que la recurrencia de episodios violentos en el marco de la práctica deportiva, se produce debido a una creciente importancia del triunfo sobre la participación, o, en términos del autor, al "ascenso de la meta del éxito" (1992, p. 279). De esta manera, la búsqueda de la victoria como único resultado aceptable promovería el aumento de la agresión de tipo instrumental.

En la tipología de la violencia humana pensada por Dunning, el tipo instrumental se refiere al empleo de esta "como medio para asegurar el logro de un fin determinado" (1992, p. 274). Esto significa que, conforme los deportes se hacen más complejos en su profesionalización, los oponentes pueden recurrir -de manera racional y calculada- a "jugar con rudeza dentro de las reglas y a recurrir a la violencia indebida con tal de lograr el éxito" (Dunning, 1992, p. 279).

Para el sociólogo británico, la violencia instrumental se contrapone a la afectiva. La primera es un modo racional y evolucionado de emplearla para la obtención de algún beneficio; en cambio, cuando esta es "tomada como un fin en sí misma" (Dunning, 1992, p. 274), es decir, cuando su uso no está ligado a la búsqueda específica de algo (o el propósito que se persigue no es considerado legítimo), él habla de una violencia "emocionalmente satisfactoria y agradable" (Dunning, 1992, p. 274).

6 Con la intención de diferenciar el deporte-educación del deporte-espectáculo, Crisorio sostiene: "es preciso ser más estricto, más meticuloso, sobre todo si se trata el deporte desde un punto de vista educativo, porque su historia, aunque marcada por los grandes acontecimientos de la historia política y económica, es relativamente autónoma" (2010, p. 189). 
Desde esta perspectiva, el problema de la violencia en los deportes radicaría en su uso racional. Mientras la afectiva -propia de las sociedades no desarrolladas- decrece conforme avanza la civilización, comienzan a surgir otros modos más elaborados, dirigidos a alcanzar un fin determinado y asociados a ciertos lazos sociales funcionales.

El creciente profesionalismo que se evidencia en deportes como el rugby, el fútbol o el boxeo se vincula estrechamente con el desarrollo de una sociedad altamente competitiva, en la cual una "elaborada división del trabajo genera asimismo una ideología en torno al éxito (...). Esta intensificación de la competitividad conduce al aumento generalizado de la rivalidad y la agresividad" (Dunning, 1992, p. 287).

A diferencia del deporte practicado en contextos escolares, donde, de acuerdo a Meirieu (2008), la violencia siempre debería ser prohibida al mismo tiempo que reelaborada positivamente, el deporte-espectáculo en ocasiones la admite -y la exacerba-como una parte más del show; el fútbol argentino de primera división constituye un claro ejemplo.

Transpolar y generalizar estas imágenes de forma indiscriminada sobre todo lo que representa el deporte (incluyendo el escolar) constituye, en cierto modo, la reproducción de un discurso irreflexivo que acaba por desatender la potencia socializadora de este tipo de prácticas. Entre los defensores de esta actividad como experiencia tendiente a la simbolización, la pacificación social y la regulación de la violencia, se destaca la perspectiva de Crisorio, quien sostiene que:

Una de las particularidades de los deportes, relacionada en parte con la institucionalización de las reglas, es, precisamente y al contrario de lo que piensa mucha gente, el control y la disminución manifiesta de la violencia física. [...] La transmisión de las reglas del juego social en todos sus aspectos y dimensiones es un objeto principal de la educación general [...], a la que la enseñanza de los deportes puede contribuir casi como ningún otro contenido de nuestra cultura. (2010, p. 191)

Como se afirmó anteriormente, donde prevalece la violencia no puede desarrollarse el deporte. El problema se suscita cuando estos límites no están claros o la agresión es un modo de interacción no identificado como falta, o, siguiendo a Elias (1992), cuando los límites de la tolerancia se encuentran muy elevados. 
Ahora bien, si una sociedad -como es el caso de Argentina- pareciera convivir a diario con un problema de violencia a escala social (Bleichmar, 2008), su eclosiónen el contexto de una práctica deportiva no debería sorprendernos. No obstante, no sería coherente culpar automáticamente al deporte por ello. Siguiendo con el análisis de Crisorio:

Si una mirada retrospectiva de corta distancia nos permitiría afirmar que, por lo menos en algunos deportes, el nivel de restricción con respecto a la violencia ha disminuido, alargando esa distancia veremos que el nivel de restricción de la violencia en los deportes de hoy es aún muy alto. $\left(2010\right.$, p. 195) ${ }^{7}$

Los hechos violentos ocurridos en el marco de una práctica deportiva no ocurren por la actividad en sí, sino por una sumatoria de condicionantes sociales que posibilitan $-\mathrm{y}$ en ocasiones promueveneste tipo de reacciones. El desconocimiento o la desconsideración de pautas regulatorias, el desinterés por el otro, la fisicalización de las rivalidades, el desprestigio de las instituciones, los efectos nocivos del resentimiento y la venganza, entre otros factores, hacen que el problema de la violencia haga su aparición en espacios y en prácticas diversos.

Sin embargo, es preciso reconocer que las categorías ligadas a la violencia, producen y reproducen prejuicios y señalamientos, en ocasiones de una forma tan apresurada que, pueden volver agresivos a aquellos a quienes se pretende excluir u ocultar. Recordemos que la caracterización de una persona así no es un designio neutral, sino que arrastra una historia y una connotación cargada de preconceptos negativos y estigmatizantes (Kaplan, 2015).

A fin de no repetir este tipo de discursos, consideramos que la enseñanza de los deportes no debe presentarse como actividades para violentos, ni tampoco, reducirse a lo terapéutico o a la rehabilitación; en contraposición, recalcamos su valor educativo ligado a la posibilidad de acercar el cuerpo a la cultura.

7 Sobre la necesidad de disminuir aún más los niveles de violencia que se suelen presenciar hoy en el contexto general de la práctica deportiva, consideramos pertinente atender a las siguientes palabras de Bayce y De Boni: "estamos todos de acuerdo en que sería mejor que hubiera menos [violencia] de la que hay; y que deberíamos todos tratar de que disminuyera, tanto dentro de las canchas, entre deportistas, como entre los espectadores, en los entornos de las canchas y en la vida cotidiana en general con motivo del deporte" (2017, p. 21). 


\section{La influencia de la masculinidad en la conformación del "futbolista ideal"}

Retomando uno de los temas desarrollados por Dunning (1992), en torno al estudio sociológico del fútbol, consideramos relevante introducir el análisis de la masculinidad como una de las representaciones sociales más significativas ligadas a este deporte, ya que históricamente sirvió -y sirve- en la producción y la reproducción de cierta identidad varonil ideal.

Para ampliar, podría afirmarse que el origen mismo del fútbol está ligado a la construcción identitaria de los gentlemen ingleses, es decir, un estereotipo de hombre ligado a valores machistas y burgueses, tales como: la virilidad, la fuerza, el liderazgo y la distinción de clase, entre otros. En palabras del propio Dunning, este deporte sirve "en parte, para educar a quienes serían los líderes militares y administrativos del Imperio británico en expansión y, en parte, como medio para inculcar y expresar la hombría" (1992, p. 328).

Asimismo, pese a los avances de las políticas de género en materia deportiva, no podemos dejar de mencionar que, históricamente, el fútbol fue utilizado como recurso para reforzar el -desigual-reparto de poder entre los sexos (en favor de los hombres). En palabras del propio Dunning:

En lo que respecta a la producción y reproducción de la identidad masculina, el deporte sólo tiene una importancia secundaria. Mucho más significativas en este aspecto son, al parecer, las características estructurales de la sociedad en general que afectan al reparto proporcional de poder entre los sexos y al grado de separación entre ellos existente en la necesaria interdependencia de hombres y mujeres. (1992, p. 342)

Antes de seguir adelante con el estudio específico del fútbol, consideramos relevante precisar de qué hablamos cuando hacemos referencia a la masculinidad. Siguiendo a Scharagrodsky (2015), es posible resumir algunos presupuestos conceptuales sobre los estudios de las masculinidades a partir de los siguientes puntos: 
a. La condición masculina es una construcción cultural que se produce y reproduce socialmente y que no puede ser definida fuera de las condiciones históricas, culturales, económicas y políticas en que ese sujeto se constituye; b) existen diversos modelos de virilidad; c) esta posición está fuertemente fijada por otras dimensiones de la subjetividad humana: la clase social, la etnia, la edad, la orientación sexual, la religión, la época, el estatus socioeconómico, etc.; d) también, se encuentra en permanente cambio y es fuente de constantes disputas, no teniendo posiciones fijas.

Además, Scharagrodsky (2007), plantea que los patrones y los estilos de masculinidades presentan cuatro aspectos fuertemente interrelacionados:

1. La masculinidad asociada a la heterosexualidad: la mayor parte de las sociedades patriarcales las identifican como dos caras de la misma moneda, siendo esta última vista como la única matriz de deseo posible.

2. La masculinidad definida por oposición a la feminidad: esta se especifica más por lo que uno no es que por lo que es. La feminidad es considerada como un constructo negativo al que hay que rechazar, naciendo la identidad masculina de cierta renuncia a lo femenino.

3. La masculinidad como un organizador de la homofobia: esta idea, tomada de Kimmel (1997), plantea a la homofobia como principio organizador de nuestra definición cultural de la virilidad. Así, "el verdadero temor no es el miedo a las mujeres, sino el de ser avergonzados o humillados delante de otros hombres o de ser dominados por hombres más fuertes" (Scharagrodsky, 2007, p. 276). La homofobia, de este modo, genera mecanismos que niegan la expresión de sentimientos entre varones. Además, la masculinidad es una aprobación homosocial: la mayoría de los hombres necesitan validar su hombría por medio de la aprobación de otros compañeros.

4. La masculinidad asociada con una cierta jerarquización del cuerpo del varón: algunas partes de este aparecen como prohibidas o condenadas. El ano, por ejemplo, se convierte en la zona degradada por excelencia. Aquel al que le "rompen el culo", lo castran y lo 
feminizan. Al mismo tiempo, el pene y la capacidad de penetrar se convierten en los aspectos más valorados en la cultura patriarcal.

En la misma línea van los planteos que consideran que la subjetividad masculina se construye desde chicos alejándose de la madre (como lugar de origen) y que deberá afianzarse en tres pilares: no ser una mujer, no ser un bebé y no ser homosexual (Burín, 2009). Finalmente, como plantea Connell (1995), la condición masculina es una construcción cultural que se produce y reproduce socialmente y que no puede ser definida fuera de las condiciones históricas, económicas y políticas en que el sujeto se constituye.

Volviendo al análisis del fútbol, nos atrevemos a afirmar que en Argentina y en muchas partes del mundo esta práctica está directamente vinculada con la forma de ser hombre; de hecho, esta actividad podría considerarse como un ritual o un paso ineludible en la constitución de la identidad masculina.

Atendiendo los aportes de Dunning (1992), en lo que refiere a la conceptualización de la violencia afectiva, nos animamos a afirmar que el fútbol argentino está impregnado de "valores" y "pasiones" que, en lugar de manifestarse en el marco de un orden simbólico, habitualmente son exteriorizados de manera física, es decir, anteponiendo el cuerpo como un depósito material de las emociones.

En este contexto, la violencia no sería una trasgresión a las leyes, sino la forma natural, de ser parte de un grupo identitario con el fútbol, que no necesariamente, lo hace a través de su práctica. De hecho, el entorno de ese deporte y las actividades comúnmente asociadas con "las barras" (como los aprietes, las agresiones a los equipos visitantes y los negocios en las inmediaciones de los estadios), en ocasiones pueden atentar contra el juego, suspendiendo partidos y provocando enfrentamientos - a veces deliberados-con la policía.

Asimismo, vale detenernos en el hecho de que el fútbol está sexuado y pintado de género, con predominio masculino, aun cuando en los últimos años aparecen cada vez más mujeres apasionadas por este deporte (Tajer, 1998).

(...) Ha sido considerado como una cosa de hombres (Llopis-Goig, 2010), un escenario social varonil, de poder y violencia masculina, donde saltan al campo los rasgos más característicos 
de los arquetipos clásicos de la masculinidad hegemónica. Este es un escenario de socialización y educación donde los varones que participan -futbolistas, entrenadores, espectadores, aficionados y medios de comunicación-, comparten una experiencia común que les permite afianzar su identidad masculina o conformar nuevas identidades". (Martín y García, 2011, p. 87)

Hay una manera de ser varón en nuestro país, que se expresa en una distintiva manera de jugar al fútbol, que ha ido cambiando con el tiempo. Se utiliza este deporte como forma de ver el mundo, como código para referirse a diversos aspectos de la vida social (Tajer, 1998).

Entonces, el fútbol argentino ha construido un tipo particular de género masculino en nuestro país y viceversa: el modo de ser hombre construye un modo de ver y jugar este deporte. Desde principios de siglo XX, en Argentina el potrero se constituye en un mundo de pibes traviesos, pícaros y "vivos", que escapan de los colegios y los clubes (Archetti, 2001).

\section{La práctica del "aguante" versus la práctica del fútbol}

Adentrándonos en el campo de los estudios sociales sobre el fútbol en Argentina, identificamos algunos referentes cuyas investigaciones hoy son consideradas fuentes ineludibles de información con respecto a la temática planteada. Entre ellos se destacan las de Alabarces (2006), Garriga (2016), Moreira (2008) y Zambaglione (2008), entre otros. Ellos coinciden en un punto nodal, la identificación del "aguante" como un aspecto central en la dinámica de aquellas hinchadas que, a través de medios debatibles tales como la extorsión, la amenaza y la violencia, persiguen intereses ajenos a dicha práctica.

Parafraseando a Alabarces (2006), el "aguante" es un término nativo, aparecido en la cultura futbolística argentina hacia comienzos de los años ochenta, que en los últimos años refiere a la puesta en acción del cuerpo, más precisamente, a la violencia física. Recuperando uno de los testimonios rastreados en una de sus investigaciones, "el aguante es demostrarle al que otro que vos tenés huevos y que te la bancás, que sos macho" (Alabarces, 2006, p. 1).

Antes de seguir adelante, consideramos necesario reiterar la diferenciación entre la práctica del fútbol y la dinámica de las hinchadas. Es 
decir, una cosa es lo que sucede dentro del campo de juego y otra lo que acontece con algunas agrupaciones conformadas en torno a algún club.

Pese a compartir entre sí ciertos valores y códigos, se trata de prácticas que tienen actores y finalidades claramente diferenciadas: mientras que los primeros buscan el éxito a partir del rendimiento y de la obtención de resultados y campeonatos en el campo de juego, los segundos intentan obtenerlo poniendo el cuerpo en el campo de batalla, o, en sus propios términos "haciendo el aguante".

Entonces, Alabarces, Garriga y Moreira (2008), proponen entender al aguante como una forma típica de honor que contempla conductas y ritos específicamente "barrabravas". De este modo, "la persona que encarna exitosamente los ideales de la barra se hace acreedora de una recompensa moral que se traduce en términos de prestigios, fama, reputación u honor" (Alabarces, Garriga y Moreira, 2008, p. 122).

En relación con las acciones consideradas violentas (por agentes externos), creemos que el uso de este tipo de recursos conlleva cierta consagración dentro del grupo de referencia, ya que poner en riesgo la propia integridad física, también puede leerse como la realización de una prueba material que confirma el compromiso y la entrega con el grupo.

\section{La enseñanza del fútbol en la escuela argentina}

En este apartado nos interesa, por un lado, indagar el modo en que el fútbol históricamente, fue considerado, en la escuela argentina y, por otro, abordar el caso de una escuela secundaria de la provincia de Buenos Aires, en relación con el modo en que se piensa y enseña este deporte en el marco de la asignatura Educación Física.

Se trata de una escuela secundaria de gestión privada, no subvencionada, laica y mixta, ubicada en una zona céntrica de la ciudad de La Plata. La cual recibe alumnos de sectores medios-altos de la sociedad. Entre los objetivos que se plantea, se destacan las ideas de "aprender a aprender", desarrollando capacidades que puedan ser aplicadas en futuras situaciones de enseñanza, y de "aprender a vivir", generando actitudes que conduzcan al alumno a ser feliz consigo mismo y con los demás.

En primer lugar, encontramos que muchas veces el fútbol está ausente en la escuela como saber por transmitir (Gutermann, 1996). 
Paradójicamente, esto sucede pese al lugar que ocupa en nuestro país como fenómeno sociocultural y atracción de masas. Siguiendo a Gutermann:

El fútbol está estrechamente vinculado a nuestra identidad nacional y atraviesa, de alguna $\mathrm{u}$ otra manera todos los sectores sociales. El fútbol no es un fenómeno «más» en nuestra realidad cotidiana, y sus formas de manifestación motriz no son neutras ni tienen el mismo valor para las personas que otro tipo de fenómeno vinculado al movimiento. (1996, p. 1)

De acuerdo con el autor recién citado, entendemos que la deliberada omisión del fútbol en la escuela se produce porque algunos docentes dan por hecho que los alumnos argentinos (se invisibiliza a las alumnas), naturalmente, saben jugar muy bien este deporte, por haberlo aprendido fuera de la institución, y que lo hacen regularmente.

En segundo orden, en nuestra experiencia como docentes de Educación Física en escuelas primarias y secundarias, nos encontramos con directivos reticentes a aceptar la inclusión de este deporte en la instancia curricular. En esos casos, se alude a cierto temor a que el fútbol genere problemas de conductas en los jóvenes, ya que estos podrían apasionarse y, en consecuencia, violentarse. Como si la agresión fuese una parte esencial del fútbol, un componente ineludible de la práctica.

Aunque suene un poco generalista, podría decirse que el fútbol ha tenido una relación contradictoria con la disciplina de la Educación Física. Dicho deporte es una de las tantas renuncias históricas de esa materia en Argentina, ya que, por ejemplo, se lo excluyó de los planes de estudio de instituciones de formación docente en esta área.

Durante años se menospreció tenazmente al fútbol, en especial porque forma parte de un capital simbólico propio de sectores populares de nuestro país; y nuestra profesión otrora (y actualmente) elitista desatendió sistemáticamente todo aquello que no fuera de origen sueco, francés o alemán (Gutermann, 1996).

Por ejemplo, en la escuela seleccionada el fútbol está presente, pero, no como contenido a ser enseñado, sino como un premio al final de la clase por cumplir con las consignas o por portarse bien. Así, el docente abandona la enseñanza de este deporte y propone simplemente un juego libre desprovisto de toda intencionalidad educativa. 
Esta situación no se produce en otros países como Estados Unidos con sus deportes más populares (como el básquetbol, el béisbol y el fútbol americano), actividades que, aunque que se practican masivamente, están incluidos en el sistema educativo y son enseñados por profesionales formados académicamente (Gutermann, 1996).

Con respecto a la enseñanza de la Educación Física en el colegio mencionado, se lleva a cabo organizando grupos de chicos y de chicas por separado. En la gran mayoría de los casos, los profesores les dan clases a los alumnos y las profesoras a las alumnas. En relación con ese modo de organizar a las personas estudiantes, hay diferentes posturas entre docentes.

Algunos consideran que ya debe superarse dicha separación, ya que no tendría razón de ser por la inexistencia de diferencias entre género a la hora de la enseñanza, mientras que otros estiman que por cuestiones biológicas (los chicos serían naturalmente más fuertes y hábiles) y de rendimiento, sería inadecuado modificarla.

De esta manera, la separación se produce exclusivamente, para la enseñanza de la materia en cuestión, no presentándose una división similar para el caso de ninguna otra asignatura. En ese contexto, de forma recreativa, dentro de Educación Física se les permite a los varones jugar al fútbol, mientras que las mujeres no cuentan con una práctica análoga.

Entonces, es posible afirmar que en las clases de fútbol se genera un clima distinto al de las demás asignaturas, llevándose a cabo una exaltación, una exacerbación, de las masculinidades de los alumnos. Dichos momentos están dados por la presencia de un conjunto de rituales (entendidos como una serie de acciones que tienen un valor simbólico y se vinculan con las tradiciones) como la utilización de botines y las charlas previas al traslado al campo de deportes (que incluyen desafíos a profesores y posibles conformaciones de equipos).

Así, se trata de una instancia que rompe con la cotidianeidad escolar, en la que se les permite exhibir a los varones comportamientos que en los demás espacios y tiempos escolares (incluida la clase convencional de Educación Física) están explícitamente prohibidos, e incluso son considerados faltas graves que acarrearían sanciones disciplinarias, nos referimos a acciones como insultar, realizar gestos obscenos, y escupir.

De esta forma, son innumerables las ocasiones en las que, durante el partido, los alumnos, generalmente a partir de situaciones de juego desfavorables o que consideran que pueden desembocar en 
situaciones así, utilizan lenguaje un tanto ofensivo o agresivo para comunicarse. Es decir, exhiben un comportamiento vinculado a la agresividad, en tanto característica atribuida históricamente al género masculino. Por ejemplo:

- "Para el otro lado, ¡la concha de la lora!" (Alumno de 4. año a otro del mismo grupo)

- “iSos pelotudo, sos pelotudo!” (Alumno de 4. año a otro del mismo grupo que cumple el rol de arquero y recibe un gol).

En muchas ocasiones, la ofensa se centra en indicarle al compañero que su condición de hombre está disminuida, refiriendo a una posible orientación sexual no heterosexual: “iJugá al fútbol, maricón!” (Alumno de $4 .^{\circ}$ año a otro del mismo grupo). Este tipo de señalamientos pueden vincularse con lo planteado por Devís et al. (2006), ya que los autores mencionados recalcan la hegemonía (excluyente) de la heterosexualidad como la forma normal de todas las relaciones sexuales y sociales.

En otras palabras, pareciera que la heterosexualidad solo puede definirse en torno a cierta representación de masculinidad, es decir, un modo "normal" de ser varón. En el mismo sentido, destacamos una intervención docente en la que, al referirse a un alumno de $6 .^{\circ}$ año, afirma "[nombre del estudiante varón] es muy putito" (luego aclara que lo dice en el sentido de que se queja mucho del arbitraje en los partidos de fútbol), lo que demuestra el papel protagónico ejercido por los profesores a la hora de reproducir sentidos.

En otra clase de Educación Física en la misma institución, otro docente, ante la falta de escucha de sus alumnos (que seguían practicando otros juegos no propuestos por el profesor), les sugiere que "se consigan novia". Esto nos habilita a pensar que, al menos en las clases de Educación Física observadas, se fomenta o transmite una heteronormatividad, (la heterosexualidad como lo normal o lo esperable).

Además, la agresión verbal se produce haciendo alusión a la existencia de una condición similar a la femenina: "iDedicate a jugar a otra cosa, a jugar con las Barbies!" (Alumno de 2. año a otro del mismo grupo). En sintonía con Scharagrodsky (2004), las mujeres suelen usarse como refuerzo de lo negativo o de lo ridículo, tanto en la interacción entre los alumnos como en la de docente-estudiantes. Aquí vemos cómo la masculinidad es definida por oposición a la feminidad: ser varón significa no ser mujer (Scharagrodsky, 2007). 
De esta forma, la etiqueta amenazante es la de "nena", lo que provoca el autocontrol para evitar comportamientos asociados con tal nombre. Caso contrario, se entiende como una debilidad femenina y/o como una falta o una negación de su masculinidad por parte de sus iguales (Clarke, 2002; Devís et al.; 2006, Flintoff, 1994).

En algunos casos, incluso, se llega a amenazar al compañero con golpearlo: "si no lo hacés [el gol], te cago a palos" (alumno de 2. año a otro del mismo grupo que está por patear al arco). En ese sentido, se registraron agravios como "¡no tenés huevo!" (alumno de $2 .^{\circ}$ año a un compañero), frase que aludiría a la falta de coraje en un hombre. Concluyendo, podemos afirmar que estas expresiones evidencian la producción y la reproducción de algunos valores vinculados históricamente con un tipo ideal de masculinidad, como son la fuerza, el coraje, la valentía y la agresividad.

\section{Consideraciones finales}

Llegados a este punto, estamos en condiciones de afirmar que el fútbol en sí mismo no es violento, ni machista. Estas representaciones son resultado de la influencia histórica de ciertos discursos hegemónicos que encuentran en la masividad del deporte y, más precisamente, en esta práctica, un modo de reproducción social sumamente efectivo.

Podría decirse que el carácter masivo y popular de este tipo de prácticas confirman su valor como parte fundamental de las sociedades occidentales, haciendo difícil imaginar hoy un país desprovisto de, al menos, un deporte representativo. Contradictoriamente, algunos educadores se rehúsan a considerar el fútbol como una práctica deportiva-educativa, creemos que esto sucede por efecto de la connotación negativa que acompaña la violencia que, innegablemente, rodea muchos espectáculos deportivos.

Como dijimos, esta traslación de prejuicios afecta particularmente la enseñanza del fútbol, no solo porque los estallidos o los enfrentamientos que acontecen en este tipo de eventos suelen difundirse masivamente, sino porque, de acuerdo con los detractores de esta práctica en la escuela, las manifestaciones violentas evidenciadas en los estadios podrían replicarse -con argumentos debatibles- en estos contextos.

En contraposición, y recuperando algunos aportes de Elias y Dunning (1992), concluimos que el fútbol en su contenido es sumamente valioso para su transmisión en cualquier ámbito educativo; esta 
afirmación se basa en dos argumentos: en primer lugar, este no puede admitir violencia bajo ninguna circunstancia ya que, cuando es debidamente fiscalizado, constituye un obstáculo para su práctica.

Dicho de otro modo, en tanto deporte de conjunto con reglas prefijadas, el fútbol tiende a mitigar la violencia, ya que el uso desmedido de la fuerza representa una falta, $y$, consecuentemente, una ventaja para el adversario. El segundo argumento para la consideración de su enseñanza se centra en su carácter popular, el cual, insistimos, creemos que debería reforzarse haciendo énfasis en su valor socializador, es decir, promoviendo el encuentro con el otro en el marco de una práctica que ha demostrado transgredir muchas diferencias sociales.

\section{Referencias}

Alabarces, P. (2006). Fútbol, violencia y política en la Argentina: ética, estética y retórica del aguante. Esporte \& Sociedade, 1(2), 21-33. https://www.ankulegi.org/wp-content/ uploads/2012/03/0102Alabarces.pdf

Alabarces, P., Garriga Zucal, J. y Moreira, V. (2008). El "aguante" y las hinchadas argentinas: una relación violenta. Horizontes Antropológicos, 14(30), 113-136. Recuperado de http://www.scielo.br/ scielo.php?script $=$ sci_arttext\&pid=S0104-71832008000200005

Archetti, E. (2001). El potrero, la pista y el ring. Las patrias del deporte argentino. Buenos Aires: Fondo de Cultura Económica.

Barbero, J. (1993) Introducción. En J. Brohm y P. Bourdieu (eds.), Materiales de sociología del deporte (pp. 9-38). Madrid: La Piqueta.

Bayce, R. e I. De Boni (2017). Preguntas y respuestas básicas sobre violencia en el deporte. En R. Bayce y B. Pereyra (coords.), Violencia en el deporte: discursos, debates y políticas en Uruguay (pp. 16-55). Montevideo: Universidad de la República.

Bleichmar, S. (2008). Violencia social-violencia escolar. Buenos Aires: Noveduc.

Burín, M. (2009). Género masculino, trabajo y subjetividad. La Matanza, $I V(7) \mathrm{http}: / / \mathrm{www}$.estudiosmasculinidades.buap.mx/num7/ genero.html

Clarke, G. (2002). Difference Matters: Sexuality and Physical Education. En D. Penney (ed.), Gender and Physical Education: Contemporary Issues and Future Directions (pp. 41-56). London: Routledge. 
Connell, R. (1995). Masculinities. Berkeley: University of California Press.

Crisorio, R. (2010). La práctica deportiva y la educación en valores. En B. Toro y A. Tallone (eds.), Educación, valores y ciudadanía (pp. 187-200). Madrid: Organización de Estados Iberoamericanos para la Educación, la Ciencia y la Cultura.

Devís, J., Fuentes, J. y Sparkes, A. (2006). ¿Qué permanece oculto del currículum oculto? Las identidades de género y de sexualidad en la Educación Física. Revista Iberoamericana de Educación, 39, 73-90. Recuperado de https://rieoei.org/historico/documentos/ rie39a03.pdf

Dirección General de Cultura y Educación de la Provincia de Buenos Aires (2010). Diseño Curricular para la Educación Secundaria Ciclo Superior. ES4: Educación Física. La Plata: Dirección General de Cultura y Educación de la Provincia de Buenos Aires. Recuperado de http://abc.gov.ar/educacion_fisica/sites/default/ files/4to_ano_-_educacion_fisica_diseno_curricular.pdf

Dunning, E. (1992). Lazos sociales y violencia en el deporte. En N. Elias y E. Dunning (eds.), Deporte y ocio en el proceso de la civilización (pp. 271-295). Madrid: Fondo de Cultura Económica.

Elias, N. (1981). Civilización y violencia. Revista Española de Investigaciones Sociológicas, 65, 141-152. http://ih-vm-cisreis.c.mad. interhost.com/REIS/PDF/REIS_065_11.pdf

Elias, N. (1992). Un ensayo sobre el deporte y la violencia. En N. Elias y E. Dunning, Deporte y ocio en el proceso de la civilización (pp.185-213). Madrid: Fondo de Cultura Económica.

Elias, N. y Dunning, E. (1992). Deporte y ocio en el proceso de la civilización. Madrid: Fondo de Cultura Económica.

Flintoff, A. (1994). Sexism and Homophobia in Physical Education: The Challenge for Teacher Education. Physical Education Review, 17(2), 97-105.

Garriga, J. (2016). El inadmisible encanto de la violencia. Buenos Aires: Cazador de tormentas libros.

Gutermann, T. (1996). El fútbol en la escuela. Aportes para pensar un encuentro. Revista Educación Física y Deportes, 1(2). Recuperado de https://www.efdeportes.com/efd2/22tag1.htm

Hernández, N. y Carballo, C. (2002). Acerca del concepto de deporte. Alcances de su(s) significado(s). Revista Educación Física \& 
Ciencia, 6, 87-102. Recuperado de https://www.efyc.fahce.unlp. edu.ar/article/download/EFyCv06a08/5640/

Kaplan, C. (2015). Violencias en plural. Sociología de las violencias en la escuela. Buenos Aires: Miño y Dávila.

Kimmel, M. (1997). Homofobia, temor, vergüenza y silencio en la identidad masculina. En T. Valdés y J. Olavarría (eds.), Masculinidad/ es. Poder y crisis (pp. 49-62). Santiago: Isis Internacional-Flacso Chile., Ediciones de las mujeres. Recuperado de https://www. caladona.org/grups/uploads/2008/01/homofobia-temor-verguenza-y-silencio-en-la-identidad-masculina-michael-s-kimmel.pdf

Kopelovich, P. (2018). Educación Física y Escuela Secundaria: sentidos y prácticas en torno a la enseñanza de la asignatura en un colegio de la Provincia de Buenos Aires (Argentina) (Tesis de maestría no publicada). Facultad Latinoamericana de Ciencias Sociales. Recuperado de https://repositorio.flacsoandes.edu.ec/ bitstream/10469/13729/2/TFLACSO-2017PK.pdf

Martín, A. y García, A. (2011). Construyendo la masculinidad: fútbol, violencia e identidad. Revista de Investigaciones Políticas y Sociológicas, 10 (2), 73-95. https://www.usc.gal/revistas/index. php/rips/article/view/828/803

Meirieu, P. (2008). Una pedagogía para prevenir la violencia en la enseñanza. Ciclo de videoconferencias. En Observatorio argentino de violencia en las escuelas, Cátedra abierta: aportes para pensar la violencia en las escuelas (pp. 93-107). Buenos Aires: Ministerio de Educación. Recuperado de http://www.bnm.me.gov.ar/ giga1/documentos/EL000315.pdf

Moreira, V. (2008). Aguante, generosidad y política en una hinchada de fútbol argentina. Avá Revista de Antropología, 12, 79 -94. https://pdfs.semanticscholar.org/bee0/c19487928e8f6f5b103ae1fc7e72773024cd.pdf

Patierno, N. (2019). La violencia (escolar) en cuestión: un estudio en escuelas secundarias públicas de la ciudad de La Plata (Tesis doctoral no publicada). Universidad Nacional de La Plata. Recuperado de http://sedici.unlp.edu.ar/bitstream/handle/10915/74108/Documento_completo.pdf-PDFA.pdf? sequence $=1 \&$ isAllowed $=\mathrm{y}$

Scharagrodsky, P. (2015). Género. En C. Carballo (ed.), Diccionario crítico de la Educación Física académica. Rastreo y análisis de 
los debates y tensiones del campo académico de la educación fisica en Argentina (pp. 243-248). La Plata: Prometeo.

Scharagrodsky, P. (2004). Juntos, pero no revueltos. La Educación Física mixta en clave de género. Cuadernos de Pesquisa, 34(121), 59-76. Recuperado de http://www.scielo.br/pdf/cp/v34n121/ a04n121.pdf

Scharagrodsky, P. (2007). Masculinidades valuadas y devaluadas. Tensiones, límites y posibilidades en el ámbito escolar. En R. Baquero, G. Diker y G. Frigerio (eds.), Las formas de lo escolar (pp. 263-284). Buenos Aires: Del Estante Editorial.

Tajer, D. (1998). El fútbol como organizador de la masculinidad. Revista de Estudios de Género, 8, 248-268. Recuperado de http:// revistalaventana.cucsh.udg.mx/index.php/LV/article/view/380

Zambaglione, D. (2008). El aguante en el cuerpo: construcción de identidad de los hinchas de un club de fútbol argentino (Tesis de maestría no publicada). Universidad Nacional de La Plata. Recuperado de http://www.memoria.fahce.unlp.edu.ar/tesis/te.370/ te.370.pdf 